\title{
Chemical synthesis and characterisation of the complement C5 inhibitory peptide zilucoplan
}

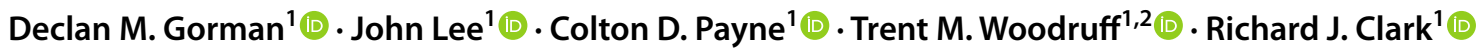

Received: 13 October 2020 / Accepted: 13 November 2020 / Published online: 4 January 2021

(c) Springer-Verlag GmbH Austria, part of Springer Nature 2021

\begin{abstract}
The complement component C5 inhibitory peptide zilucoplan is currently in phase III clinical trials for myasthenia gravis (MG). Despite being at an advanced stage of clinical development, there have been no published reports in the literature detailing its chemical synthesis. In this work, we describe an approach for the chemical synthesis of zilucoplan and validate that the synthesised compound blocks LPS-induced C5a production from human blood.
\end{abstract}

Keywords Peptides · Zilucoplan · RA101495 · Peptide synthesis · Complement · C5 · C5a

\section{Introduction}

Zilucoplan (formerly RA3193/RA101495) is a $3.5 \mathrm{kDa}$ peptide therapeutic (Fig. 1) developed by RA Pharmaceuticals targeting the complement component $\mathrm{C} 5$, with the purpose of preventing the cleavage of $\mathrm{C} 5$ into the downstream complement components C5a and C5b (Hoarty et al. 2015; Howard et al. 2020). Both metabolites are associated with host defence, with C5a propagating leukocyte chemotaxis and cytokine release through its receptors C5aR1 and C5aR2 (Pandey et al. 2020), and C5b initiating the formation of the membrane attack complex (MAC) (Denk et al. 2017; Podack 1984; Schindler et al. 1990; Scholz et al. 1990). Zilucoplan also binds to $\mathrm{C} 5 \mathrm{~b}$, thereby competitively inhibiting $\mathrm{C} 5 \mathrm{~b}$ binding to $\mathrm{C} 6$ and further preventing assembly of the MAC (Yee et al. 2015).

Zilucoplan has a similar mechanism of action to eculizumab (Soliris), an already approved therapeutic for

Handling Editor: J. D. Wade.

Electronic supplementary material The online version of this article (https://doi.org/10.1007/s00726-020-02921-5) contains supplementary material, which is available to authorized users.

Richard J. Clark

richard.clark@uq.edu.au

1 School of Biomedical Sciences, The University of Queensland, Brisbane, Australia

2 Queensland Brain Institute, The University of Queensland, Brisbane, Australia paroxysmal nocturnal hemoglobinuria, atypical hemolytic uremic syndrome, neuromyelitis optica and MG (Brodsky et al. 2008; Cofiell et al. 2015; Howard et al. 2017; Legendre et al. 2013; Pittock et al. 2013). Both bind to C5 and prevent the pro-inflammatory and potentially cell-destructive activity elicited by C5 metabolites C5a and C5b (Beecher et al. 2019).

While zilucoplan and eculizumab inhibit C5 cleavage, several therapeutics have been developed specifically targeting the downstream complement interaction of C5a-C5aR1, enabling a more specific suppression of C5a-initiated systemic inflammation. This ligand-receptor complex has been a target for the purposes of treating chronic inflammatory conditions such as inflammatory bowel disease and ANCAassociated vasculitis (Jayne et al. 2017; Woodruff et al. 2005). Recently, heightened C5a-C5aR1 interaction, and the suppression of such, has also been of interest due to its association with more severe cases of COVID-19 (Carvelli et al. 2020; Lo et al. 2020; Woodruff and Shukla 2020).

Zilucoplan is currently in phase III clinical trials for the treatment of MG following encouraging phase II trial results (Howard et al. 2020). However, despite this peptide being in advanced phase clinical trials, there is only a brief description of its synthesis presented in patent material (Hoarty et al. 2015). We, therefore, set out to undertake the chemical synthesis and experimental validation of our synthesised peptide. Here we report an approach for the solid-phase peptide synthesis of zilucoplan and verify it as a potent human C5 inhibitor. 

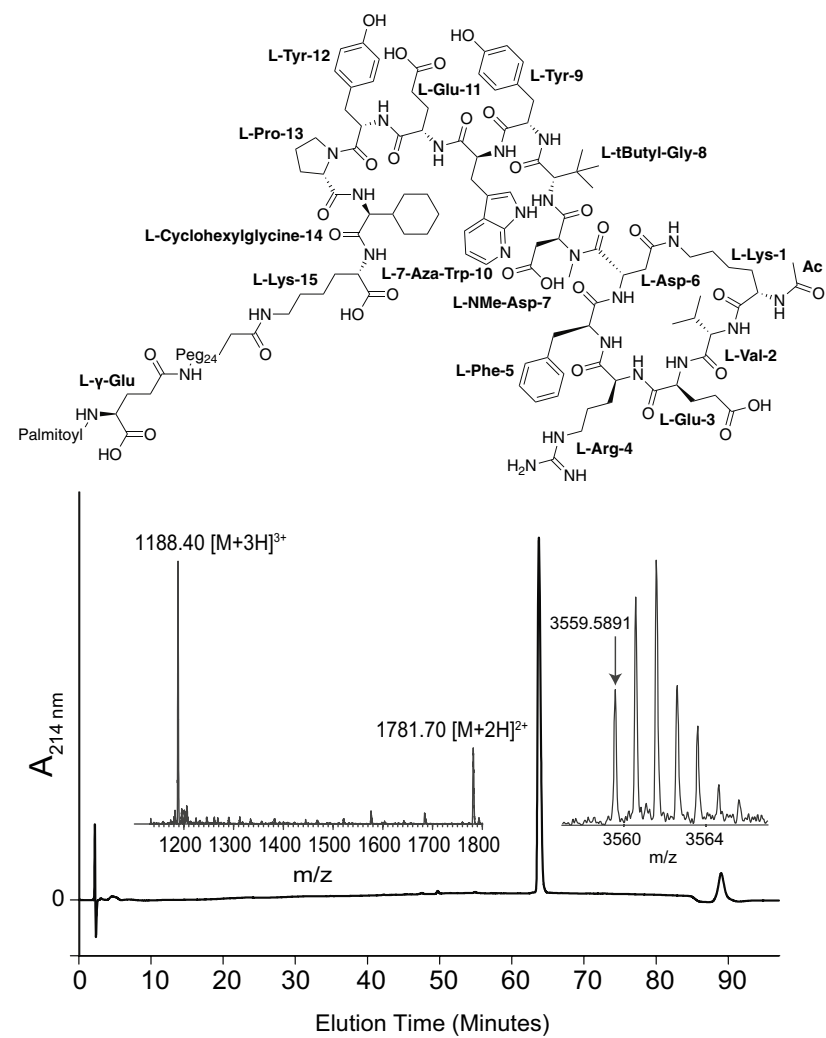

Fig. 1 Structure and analysis of synthetic zilucoplan. The structure of zilucoplan synthesized in this study (top). The RP-HPLC trace, ESI-MS spectrum and MALDI-MS spectrum of zilucoplan (bottom). RP-HPLC was performed with an increasing gradient of $1 \%$ buffer B (90\% ACN, $0.05 \%$ TFA) in buffer A (0.05\% TFA) per minute. Sample $>99 \%$ pure

\section{Methods}

\section{Peptide synthesis and isolation}

Peptides were synthesised using Fmoc (9-fluorenylmethyloxycarbonyl)-based solid-phase peptide synthesis (SPPS) on Wang resin using standard coupling protocols (Zhang et al. 2019). Four equivalents of standard amino acid, 2-(1H-benzotriazol-1-yl)-1,1,3,3-tetramethyluronium hexafluorophosphate (HBTU) and diisiopropylethylamine (DIPEA) were used for each coupling step. Non-standard amino acids used in this synthesis included Fmoc-Lysine(ivDde)-OH, Fmoc-L-cyclohexylglycine-OH, Fmoc-L-7-azatryptophan-OH, Fmoc-L-tbutyl-glycine-OH, Fmoc-N-Me-Asp(OtBu)-OH, Fmoc-Lys(Mtt)-OH, FmocAsp(O-2-PhiPr)-OH, Fmoc-Glu(OH)-OtBu and FmocNH-PEG24- $\mathrm{CH}_{2} \mathrm{CH}_{2} \mathrm{COOH}$. All non-standard amino acids were coupled twice ( $30 \mathrm{~min}$ for each reaction) using two equivalents of amino acid, two equivalents of HBTU and DIPEA in DMF.
Lactam bridge formation between Lys- 1 to Asp- 6 was achieved by selectively deprotecting the sidechains with $1 \%$ trifluoroacetic acid (TFA) in dichloromethane $(15 \times 2 \mathrm{~min})$, to remove the MTT and O-2-PhiPr protecting groups, respectively. Following deprotection, two equivalents of 1-[Bis(dimethylamino)methylene]1H-1,2,3-triazolo[4,5-b]pyridinium 3-oxide hexafluorophosphate (HATU) was dissolved in minimal dimethylformamide (DMF). HATU in DMF was added to the resin along with 15 equivalents DIPEA; resin was then shaken overnight $(\sim 16 \mathrm{~h})$. Lactam bridge formation was confirmed via test cleavage using electrospray mass spectrometry (ESI-MS) (AB SCIEX API 2000). N-terminal acetylation was then carried out on-resin with a solution of acetic anhydride (5\%) (Sigma-Aldrich) and DIPEA (3\%) (Sigma-Aldrich) in DMF at $25{ }^{\circ} \mathrm{C}$ for $2 \times 10 \mathrm{~min}$. $10 \%$ hydrazine monohydrate (Sigma-Aldrich) in DMF was used for the removal of ivDde from the sidechain of the C-terminal lysine following the synthesis of the mainchain $(2 \times 10$-min treatments). Resin was split at this point, and one half was cleaved and purified for NMR analysis of the non-PEGylated/lipidated peptide. Fmoc-Glu(OH)$\mathrm{OtBu}$ and Fmoc-PEG24- $\mathrm{CH}_{2} \mathrm{CH}_{2} \mathrm{COOH}$ were then coupled sequentially to the remaining resin.

Following synthesis, the peptides (with and without PEG group/lipid) were cleaved off resin and side chain protecting groups were removed by treatment with trifluoroacetic acid/TIPS/ $\mathrm{H}_{2} \mathrm{O}$ at a ratio of $95: 2.5: 2.5$ for $2 \mathrm{~h}$ at room temperature. The peptide was then purified using reverse phase high-performance liquid chromatography (RP-HPLC) with an increasing gradient of $1 \%$ buffer B (90\% ACN, $0.05 \%$ TFA) in buffer A $(0.05 \%$ TFA) per minute. HPLC fractions were analysed by electrospray mass spectrometry (ESI-MS) and fractions containing the desired product were combined. Peptide was purified until $>99 \%$ purity as assessed by analytical RP-HPLC (Agilent, $5 \mu \mathrm{M}, 2.1 \times 150 \mathrm{~mm}$ ). Pure peptide was analysed by high-resolution mass spectrometry using MALDI-TOF MS (AB SCIEX TOF/TOF-5800).

Palmitic acid-NHS ester (Sigma-Aldrich) was used to label the free amine of the PEGylated peptide off-resin. Palmitic acid-NHS ester and peptide were prepared as stock solutions in DMSO at a concentration of $10 \mathrm{mg} /$ $\mathrm{ml}$ and $2.5 \mathrm{mg} / \mathrm{ml}$, respectively. NHS ester and peptide solutions were then combined at a ratio of 5:1 (NHS ester:peptide) and triethylamine (TEA) in DMSO was then added (two equivalents relative to peptide). The solution was stirred for $16 \mathrm{~h}$ before confirming attachment of palmitic acid by ESI-MS. The peptide was purified using RP-HPLC, fractions were lyophilised and confirmed $>99 \%$ pure via analytical RP-HPLC (Agilent). $12 \mathrm{mg}$ of pure peptide was obtained following synthesis, representing a 
yield of $2.7 \%$ of the theoretical maximum based on resin loading.

\section{Nuclear magnetic resonance (NMR)}

Zilucoplan was prepared for NMR analysis by dissolving $1 \mathrm{mg}$ of peptide in $550 \mu \mathrm{l}$ of $\mathrm{H}_{2} \mathrm{O} / \mathrm{D}_{2} \mathrm{O}(90: 10)$, at $\mathrm{pH} \sim 3.5$. Two-Dimensional ${ }^{1} \mathrm{H}-{ }^{1} \mathrm{H}$ TOCSY and NOESY experiments were recorded, with mixing times of 80 and $200 \mathrm{~ms}$ respectively, at $298 \mathrm{~K}$ on a $900 \mathrm{MHz}$ Bruker Avance III Spectrometer equipped with a cryoprobe (Braunschweiler and Ernst 1983; Jeener et al. 1979). The data were processed using Topspin 4.0.3 (Bruker), with the spectra being referenced to the solvent signal at $4.77 \mathrm{ppm}$. Spectra were assigned using sequential assignment strategies with the program CARA (Keller 2004; Wüthrich 1986). Secondary structure was identified via the determination of secondary $\mathrm{H} \alpha$ shifts from the closest naturally occurring residues random coil values (Wishart et al. 1995).

\section{Ex vivo human whole blood assay}

Fresh human blood was collected from healthy volunteers with informed consent. Blood was collected using anticoagulant lepirudin at $50 \mu \mathrm{g} / \mathrm{mL}$. Blood samples were pretreated with zilucoplan at ranging doses of $1 \mathrm{nM}$ to $1 \mu \mathrm{M}$ for $30 \mathrm{~min}$ at $37^{\circ} \mathrm{C}$. Lipopolysaccharides from Echerichia coli $\mathrm{O} 111: \mathrm{B} 4$ at $200 \mathrm{ng} / \mathrm{mL}$ was added to the pre-incubated blood samples for $24 \mathrm{~h}$ at $37^{\circ} \mathrm{C}$. Blood samples were centrifuged at $2000 \times g$ for $10 \mathrm{~min}$ at $4{ }^{\circ} \mathrm{C}$. Plasma C5a levels were determined using commercially available enzyme-linked immunosorbent assay kit (R\&D Systems).

\section{Results}

We employed the structure of zilucoplan described in patent material for the basis of this work (Fig. 1) (Hoarty et al. 2015). Zilucoplan consists of a cyclic backbone (from the sidechain of Lys-1 to the sidechain of Asp-6) alongside several non-natural amino acids (including cyclohexyl glycine, L-7-azatryptophan, L-butyl-glycine and N-methyl aspartate). PEG24, $\gamma$-glutamic acid and an N-terminal palmitic acid moiety were attached to the sidechain of Lys- 15 . The cyclic core of zilucoplan was synthesised first using solid-phase peptide synthesis. The $\mathrm{N}$-terminus was acetylated and then an on-resin cyclisation was performed between the sidechain of Lys-1 and Asp- 6 overnight. The reaction was confirmed as completed by a loss of 18 Da observed with ESI-MS. Subsequent removal of the IvDde group on Lys-15 sidechain facilitated addition of PEG24 and $\gamma$-glutamic acid to this position.

Following 95\% TFA cleavage and RP-HPLC purification, palmitic acid was coupled onto the amine of $\gamma$-glutamic acid on
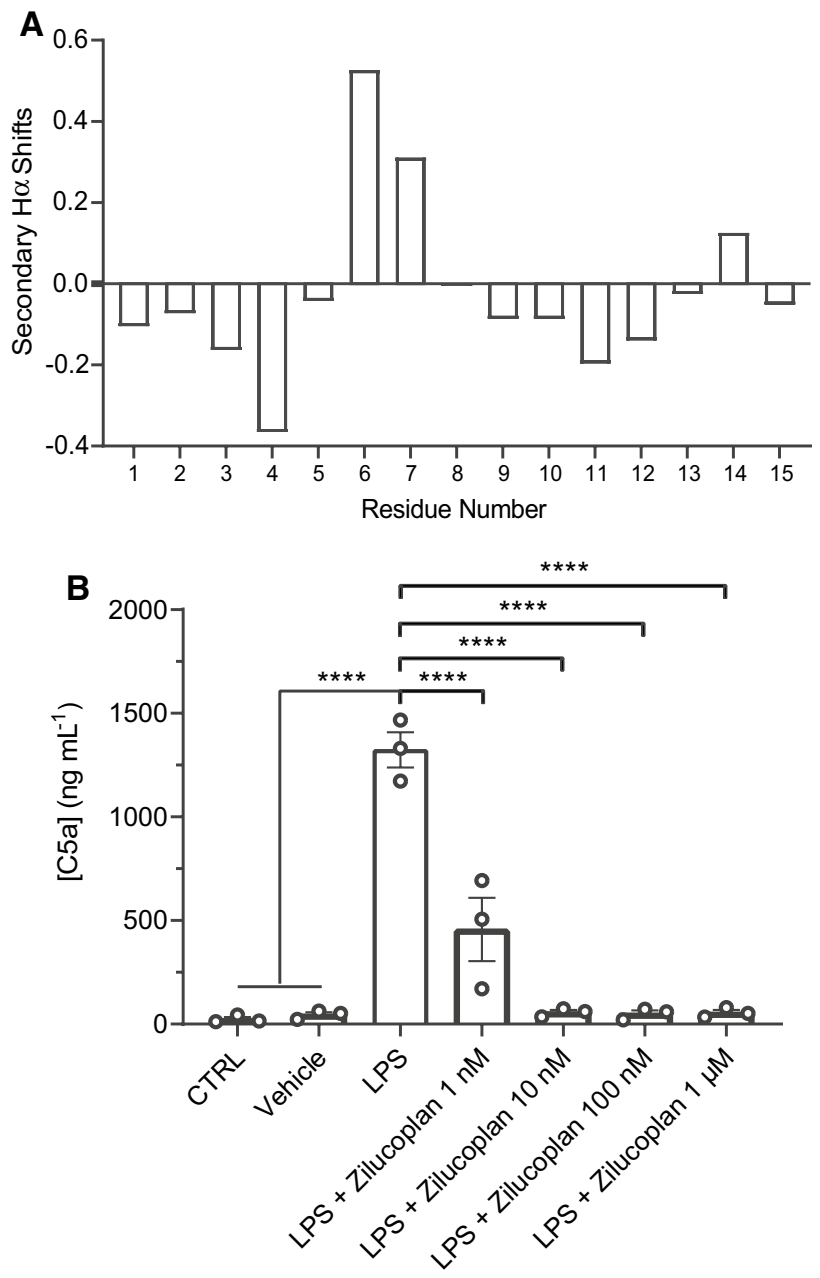

Fig. 2 Structural analysis and biological activity of synthesized zilucoplan. a Calculated secondary $\mathrm{H} \alpha$ shifts of the main chain of zilucoplan. Random coil values from the closest naturally occurring amino acid were used for nonstandard residues. b LPS-induced plasma C5a levels of human whole blood. $n=3$, data displayed as a mean \pm SEM. $* * * * p<0.0001$

the sidechain extension of Lys-15 utilising palmitic acid-NHS ester. After purification by RP-HPLC, $12 \mathrm{mg}$ of zilucoplan was obtained ( $>99 \%$ purity, $2.70 \%$ yield based on resin loading) and the monoisotopic mass (observed $3559.5891 \mathrm{Da}$, calculated $3559.9694 \mathrm{Da}$ ) was consistent with the reported value (Fig. 1) (Hoarty et al. 2015). Secondary H $\alpha$ shifts were calculated according to the chemical shift assignments (Table S1) of the main chain of zilucoplan (not including PEG24, $\gamma$-glutamic acid and palmitic acid). The deviation from random coil between residues 4 and 7 suggests that there might be local rigidity between Arg-4 and NMe-Asp-7 (Fig. 2a).

The ability of zilucoplan to inhibit LPS-induced increase in C5a plasma levels in human whole blood was then determined in a concentration-response assay. Complete inhibition of LPS-induced C5a levels were observed at zilucoplan concentrations ranging from $10 \mathrm{nM}$ to $1 \mu \mathrm{M}$, with a $65.7 \%$ 
reduction in C5a plasma levels observed at a concentration of $1 \mathrm{nM}$ (Fig. 2b). An $\mathrm{IC}_{50}$ of the synthesised zilucoplan was $474.5 \mathrm{pM}$.

\section{Discussion}

Prior to this work, there has been only a brief description of the synthesis of zilucoplan in the patent literature (Duda et al. 2020; Hoarty et al. 2015; Ma et al. 2020). Aside from being a promising therapeutic, zilucoplan is also a useful research tool in the study of complement component C5 activities. In this study, we have described an effective synthesis protocol for zilucoplan and validated the efficacy/ potency of the resulting product. It has been previously reported that zilucoplan has little to no effect on rodent C5 cleavage, but does bind and inhibit human C5 cleavage (Hoarty et al. 2015; Ricardo et al. 2017). Consequently, as a measure of C5 cleavage inhibition, an LPS-induced C5a generation assay in whole human blood was assessed. Here we demonstrated a comparable inhibition of C5a formation with our synthesis compound $\left(\mathrm{IC}_{50}=474.5 \mathrm{pM}\right)$, to the zilucoplan compound described in the patent literature $\left(\mathrm{IC}_{50}=4.8 \mathrm{nM}\right)$ (Ricardo et al. 2017).

In conclusion, our effective synthesis of zilucoplan and its validation as a potent inhibitor of C5a production will be valuable to the research community to facilitate future studies on the complement system and for use as a benchmark molecule for future drug discovery efforts.

Author contributions All authors contributed to the study conception and design. Material preparation, data collection and analysis were performed by DMG, JL and CDP. All authors contributed to the writing and revisions of the manuscript.

Funding This work was funded, in part, by a National Health and Medical Research Council Development Grant (APP8881111 to TMW and RJC).

Data availability The datasets generated during and/or analysed during the current study are available from the corresponding author on reasonable request.

\section{Compliance with ethical standards}

Conflict of interest The authors declare that they have no conflict of interest.

Ethical approval Not applicable.

\section{References}

Beecher G, Putko BN, Wagner AN, Siddiqi ZA (2019) Therapies directed against B-cells and downstream effectors in generalized autoimmune myasthenia gravis: current status. Drugs 79:353364. https://doi.org/10.1007/s40265-019-1065-0

Braunschweiler L, Ernst RR (1969) Coherence transfer by isotropic mixing: Application to proton correlation spectroscopy. J Magn Reson 53:521-528. https://doi.org/10.1016/00222364(83)90226-3

Brodsky RA et al (2008) Multicenter phase 3 study of the complement inhibitor eculizumab for the treatment of patients with paroxysmal nocturnal hemoglobinuria. Blood 111:1840-1847. https://doi.org/10.1182/blood-2007-06-094136

Carvelli $\mathbf{J}$ et al (2020) Association of COVID-19 inflammation with activation of the $\mathrm{C} 5 \mathrm{a}-\mathrm{C} 5 \mathrm{aR} 1$ axis. Nature. https://doi. org/10.1038/s41586-020-2600-6

Cofiell R et al (2015) Eculizumab reduces complement activation, inflammation, endothelial damage, thrombosis, and renal injury markers in aHUS. Blood 125:3253-3262. https://doi. org/10.1182/blood-2014-09-600411

Denk S et al (2017) Complement C5a-induced changes in neutrophil morphology during inflammation. Scand J Immunol 86:143155. https://doi.org/10.1111/sji.12580

Duda P, Farzaneh-Far R, Ma Z, Zhu N, Thackaberry E, Ricardo A (2020) Neurological disease treatment with zilucoplan. WO2020086506A1

Hoarty MD et al. (2015) Modulation of complement activity. United States of America Patent, WO2015191951A2

Howard JF Jr et al (2017) Safety and efficacy of eculizumab in antiacetylcholine receptor antibody-positive refractory generalised myasthenia gravis (REGAIN): a phase 3, randomised, doubleblind, placebo-controlled, multicentre study. Lancet Neurol 16:976-986. https://doi.org/10.1016/s1474-4422(17)30369-1

Howard JF Jr et al (2020) Clinical effects of the self-administered subcutaneous complement inhibitor zilucoplan in patients with moderate to severe generalized myasthenia gravis: results of a phase 2 randomized double-blind, placebo-controlled, multicenter clinical trial. JAMA Neurol 77:582-592. https://doi. org/10.1001/jamaneurol.2019.5125

Jayne DRW et al (2017) Randomized trial of C5a receptor inhibitor avacopan in ANCA-associated vasculitis. J Am Soc Nephrol 28:2756. https://doi.org/10.1681/ASN.2016111179

Jeener J, Meier BH, Bachmann P, Ernst RR (1979) Investigation of exchange processes by two-dimensional NMR spectroscopy. J Chem Phys 71:4546-4553. https://doi.org/10.1063/1.438208

Keller R (2004) The computer aided resonance assignment tutorial. CANTINA, Seattle

Legendre CM et al (2013) Terminal complement inhibitor eculizumab in atypical hemolytic-uremic syndrome. N Engl J Med 368:2169-2181. https://doi.org/10.1056/NEJMoa1208981

Lo MW, Kemper C, Woodruff TM (2020) COVID-19: complement, coagulation, and collateral damage. J Immunol. https://doi. org/10.4049/jimmunol.2000644

Ma Z, Zhu N, Thackaberry E, Farzaneh-Far R, Ricardo A (2020) Modulation of complement activity. WO2020185541

Pandey S, Maharana J, Li XX, Woodruff TM, Shukla AK (2020) Emerging insights into the structure and function of complement C5a receptors. Trends Biochem Sci 45:693-705. https:// doi.org/10.1016/j.tibs.2020.04.004

Pittock SJ et al (2013) Eculizumab in AQP4-IgG-positive relapsing neuromyelitis optica spectrum disorders: an open-label pilot study. Lancet Neurol 12:554-562. https://doi.org/10.1016/s1474 $-4422(13) 70076-0$ 
Podack ER (1984) Molecular composition of the tubular structure of the membrane attack complex of complement. J Biol Chem 259:8641-8647

Ricardo A et al. (2017) Modulation of complement activity. United States of America Patent, WO2018106859 Al

Schindler R, Gelfand JA, Dinarello CA (1990) Recombinant C5a stimulates transcription rather than translation of interleukin-1 (IL-1) and tumor necrosis factor: translational signal provided by lipopolysaccharide or IL-1 itself. Blood 76:1631-1638

Scholz W, McClurg MR, Cardenas GJ, Smith M, Noonan DJ, Hugli TE, Morgan EL (1990) C5a-mediated release of interleukin 6 by human monocytes. Clin Immunol Immunopathol 57:297-307. https://doi.org/10.1016/0090-1229(90)90043-p

Wishart DS, Bigam CG, Holm A, Hodges RS, Sykes BD (1995) 1H, $13 \mathrm{C}$ and $15 \mathrm{~N}$ random coil NMR chemical shifts of the common amino acids. I. Investigations of nearest-neighbor effects. J Biomol NMR 5:67-81. https://doi.org/10.1007/BF00227471

Woodruff TM, Shukla AK (2020) The complement C5a-C5aR1 GPCR axis in COVID-19 therapeutics. Trends Immunol. https://doi. org/10.1016/j.it.2020.09.008
Woodruff TM et al (2005) Increased potency of a novel complement factor 5a receptor antagonist in a rat model of inflammatory bowel disease. J Pharmacol Exp Ther 314:811-817. https://doi. org/10.1124/jpet.105.086835

Wüthrich K (1986) NMR with proteins and nucleic acids. Europhys News 17:11-13

Yee A et al (2015) Visualization of an N-terminal fragment of von Willebrand factor in complex with factor VIII. Blood 126:939942. https://doi.org/10.1182/blood-2015-04-641696

Zhang J et al (2019) An ancient peptide family buried within vicilin precursors. ACS Chem Biol 14:979-993. https://doi.org/10.1021/ acschembio.9b00167

Publisher's Note Springer Nature remains neutral with regard to jurisdictional claims in published maps and institutional affiliations. 